\title{
Autoría de regalo: una aproximación a su frecuencia en una revista peruana
}

\author{
Jessica Hanae Zafra-Tanaka1, Cristian Roca², Jorge Luis Cañari-Casaño², Ana \\ Vargas-Calla ${ }^{3}$ \\ ${ }^{1}$ Centro de Excelencia en Enfermedades Crónicas, Universidad Peruana Cayetano Heredia, \\ Lima, Perú \\ ${ }^{2}$ Facultad de Salud Pública, Universidad Peruana Cayetano Heredia, Lima, Perú \\ ${ }^{3}$ Facultad de Medicina Veterinaria, Universidad Nacional Mayor de San Marcos, Lima, Perú
}

Introducción. La autoría injustificada o 'autoría de regalo' es una práctica inadecuada que consiste en nombrar como autores a personas que no cumplen los criterios de autoría. Los informes de investigaciones científicas suelen ser publicados como artículos originales en revistas científicas y pueden presentar estas prácticas inadecuadas.

Objetivos. Determinar la prevalencia de autoría de regalo en publicaciones de artículos originales.

Materiales y métodos. Se trata de un estudio descriptivo en el cual se revisó la sección de contribuciones de autoría de todos los artículos publicados en una revista peruana desde enero de 2013 hasta marzo de 2017. Se consideró una autoría de regalo cuando un autor no cumplía con, al menos, uno de los criterios establecidos por el International Committee of Medical Journal Editors (ICMJE).

Resultados. De los 209 artículos originales publicados, 11 fueron excluidos debido a que no reportaron las contribuciones de autoría. La prevalencia de autoría de regalo de los 198 artículos incluidos fue de 106 (53,5\%). Los criterios que menos cumplieron fueron la aprobación final del manuscrito (23,2 \%), y su redacción y revisión crítica (16,8 \%).

Conclusiones. Es necesario que las instituciones educativas capaciten a los investigadores para que discriminen entre autoría y contribución. Además, es necesario que las revistas soliciten y corroboren las contribuciones reportadas.

Recibido: $02 / 02 / 18$

Aceptado: $20 / 09 / 18$

Publicado: 25/09/18

Citación:

Zafra-Tanaka JH, Roca C, Canari-Casano JL,

Vargas-Calla A. Autoría de regalo: una aproximación a su frecuencia en una revista peruana. Biomédica. 2019;39:323-9.

https://doi.org/10.7705/biomedica.v39i3.4316

\section{Correspondencia:}

Jessica Hanae Zafra-Tanaka, Avenida

Conquistadores $N^{\circ} 365$, San Isidro Lima, Perú

Teléfono: (51) (99) 4371991

j.zafra.t@gmail.com

Contribución de los autores:

Todos los autores participaron en el diseño de estudio, la recolección de datos, el análisis y la interpretación de los resultados, y en la redacción del manuscrito.

\section{Financiación:}

El presente estudio no recibió ninguna subvención específica de ninguna agencia de financiamiento. Jessica Hanae Zafra-Tanaka recibió apoyo financiero de CRONICAS, Centro de Excelencia en Enfermedades Crónicas a través de la beca CRONICAS 2017. Cristian Roca recibió capacitación en el programa de entrenamiento "Infectious Diseases Training Program in Bolivia: South-South Training with Perú" NIH/Fogarty (5D43TW010074-04). Ana Vargas Calla recibió capacitación en el programa "Training in Infectious Diseases in Perú - Time for Capacity Strengthening in Clinical Research" (5D43TW001140).

Conflicto de intereses:

Los investigadores no tienen conflictos de intereses que declarar.
Palabras clave: autoría; escritura; artículo de revista; bibliometría; Perú.

\section{Gift authorship: An approach to its frequency in a Peruvian journal}

Introduction: Unjustified authorship or "gift authorship" is an inadequate practice of authorship that consists of naming as authors people who do not meet the authorship criteria. Reports of scientific research are often published as original articles in scientific journals and may present these inappropriate practices.

Objective: Determine the prevalence of gift authorship in original articles for publication. Materials and methods:. Descriptive study in which the authorship contributions section of all the articles published between 2013 and the first quarter of 2017 in a Peruvian magazine was reviewed. Gift authorship was considered when an author did not meet at least one of the criteria established by the International Committee of Medical Journal Editors (ICMJE). Results: Of the 209 original articles published, 11 were excluded because they did not report authorship contributions. The prevalence of gift authorship was 53.5\% (106). The critreria least met were the final approval of the manuscript (23.2\%) and the writing and critical review of this manuscript. (16.8\%).

Conclusions: It is necessary that educational institutions train researchers to distinguish between authorship and contribution. In addition, it is necessary that the journals request and corroborate the reported contributions.

Keywords: authorship, writing; journal article; bibliometrics; Perú.

La asignación correcta de las autorías en el informe de una investigación científica es importante porque refleja un logro académico e implica el financiamiento y la responsabilidad sobre lo publicado $(1,2)$. Estos informes suelen ser publicados en formatos de artículos originales en las revistas científicas. En el 2013, el International Committee of Medical Journal Editors (ICMJE) planteó los criterios que se deben cumplir para ser considerado como autor de una publicación científica, los cuales se actualizaron en el $2017(1,3)$. En ambos se definió a un autor como aquella persona que hace contribuciones substanciales al trabajo de investigación (diseño del estudio, adquisición, análisis o interpretación de los datos), participa de la redacción del manuscrito, 
aprueba su versión final, y acepta responsabilidad sobre la precisión e integridad de lo que se publica. Además, se hizo la distinción entre autores, es decir, aquellos que cumplen con los criterios, y contribuyentes, aquellas personas que hicieron algún aporte sin llegar a cumplir con todos (1).

Durante el proceso de investigación científica los investigadores pueden incurrir en prácticas inadecuadas de autoría. Si bien existen diversas maneras de clasificar las prácticas inadecuadas de autoría, estas se podrían dividir en la autoría injustificada o 'de regalo' y la autoría fantasma (4). La primera se refiere a asignarle una autoría a alguien que no cumple con los criterios de autoría (1). Esto puede deberse a un acuerdo recíproco entre autores para colocarse mutuamente en sus publicaciones o al hecho de colocar como autor a un investigador renombrado con la finalidad de aumentar el prestigio del trabajo, ganar visibilidad y pasar fácilmente la revisión por pares, o considerar como autor a alguien por respeto o gratitud $(5,6)$; también, puede deberse a miedo, intimidación, obligación o falta de conocimiento (5). La autoría fantasma, por su parte, se refiere a no asignarle una autoría a alguien que cumplió con los criterios para ser autor (4).

Se han realizado diversos estudios sobre la prevalencia de la autoría de regalo (7-10), y se han hallado prevalencias que varían de 6,0 a $89,0 \%$ en artículos originales. La autoría de regalo se observa en diversos tipos de publicaciones: artículos originales (16\%) (10), protocolos de ensayos clínicos (33\%) (8) y revisiones sistemáticas Cochrane (41\%) (9). Además, no solo se ha informado en revistas de baja difusión, sino también en revistas de alta difusión como: Annals of Internal Medicine (15,5\%), Journal of the American Medical Association (16,7 \%), New England Journal of Medicine (12,2 \%), Lancet (19,7\%), Plos One (22,8 \%) o Nature Medicine (29,3\%); la mayor proporción se presenta en los artículos originales (25\%), en comparación con las revisiones narrativas o sistemáticas (15\%) (10).

Perú incrementó el número de publicaciones científicas entre los años 2000 y 2009, según un estudio que revisó las publicaciones indizadas en ISI Web of Knowledge (11) y, teniendo en cuenta las publicaciones entre 2007 y 2016 , se encontraba en el octavo puesto en Latinoamérica según el ranking de los países elaborado por Scimago (12). La producción científica debe estar liderada por investigadores que se ciñan a las buenas prácticas y a los estándares internacionales, sin embargo, se han documentado problemas de autoría inadecuada. Así en un reporte de casos de un hospital del seguro social, se presentó un caso en el cual el jefe del servicio donde se realizó una investigación quería ser autor de la publicación sin haber participado en ella (13). Esto refleja problemas como la falta de conocimiento de los criterios de autoría, la confusión entre responsabilidad administrativa y autoría, o las exigencias inadecuadas.

Diversos problemas pueden desencadenarse debido a las prácticas inadecuadas de autoría. Por ejemplo, el caso de Andrew Wakefield ejemplifica como, de haber cumplido con sus responsabilidades, los coautores habrían revisado en detalle el contenido del artículo científico y se hubiera podido evitar la publicación de resultados fraudulentos y las consecuencias en salud pública que trajo la publicación de aquel informe (14).

Por ello, el presente estudio tuvo como objetivo estimar la frecuencia de la autoría de regalo de los artículos originales publicados desde enero de 2013 hasta marzo de 2017 en una revista peruana, según las recomendaciones establecidas por el ICMJE, con el propósito de tener una aproximación de la magnitud del problema y proponer medidas de prevención y control. 


\section{Materiales y métodos}

Se llevó a cabo un estudio descriptivo en el cual se analizó la sección de contribuciones de autoría de los artículos originales publicados en los últimos cinco años (2013-2017) en la Revista Peruana de Medicina Experimental y Salud Pública. Esta revista publica cuatro números al año, por lo cual se incluyeron todos los números publicados en los años 2013 a 2016 y el primer número publicado en el 2017. Se incluyeron todos los artículos originales, y se excluyeron las revisiones narrativas, los editoriales, las cartas al editor y los simposios. Además, se excluyeron aquellos artículos que no presentaron la sección de contribuciones de autoría.

Se eligió la Revista Peruana de Medicina Experimental y Salud Pública, ya que es la revista de salud pública más importante del Perú y una de las pocas que publican la sección de contribuciones de autoría. Desde el 2000 está indizada en Medline/Index Medicus, una de las bases de datos más rigurosas del ámbito científico a nivel mundial (15). Además, consideramos que refleja de forma adecuada las publicaciones científicas en el área de ciencias de la salud de Perú.

Para la recolección de los datos, se recuperaron todos los artículos originales desde la página web de la Revista Peruana de Medicina Experimental y Salud Pública y se diseñó una plantilla con el programa Excel $^{\mathrm{TM}}$ en la cual se recolectó la siguiente información: título del artículo, código doi, número de autores, año de publicación del artículo, número y volumen de la revista. Además, se evaluaron los criterios de autoría para cada uno de los autores de cada artículo científico. La variable numérica que se incluyó fue el número de autores y las variables categóricas fueron los criterios de autoría, analizados para cada autor, el año de publicación y la presencia de autoría de regalo.

La sección de contribuciones de los autores fue evaluada por dos revisores de forma independiente para determinar si estos cumplían con los criterios establecidos por el International Committee of Medical Journal Editors. Posteriormente, estas evaluaciones fueron contrastadas y, en caso de discordancias, se revisó nuevamente el artículo y ambos revisores lo discutieron hasta llegar a un consenso.

\section{Variables de estudio}

Se definió como autoría de regalo la inclusión como autores de quienes no cumplían con todos los criterios establecidos por el International Committee of Medical Journal Editors (1). Estos mismos criterios son requisitos establecidos para publicar artículos originales en la Revista Peruana de Medicina Experimental y Salud Pública. (16). Solo se consideró como autor a aquella persona que cumplía con todos los criterios citados a continuación:

- contribuciones significativas a la concepción o diseño del manuscrito, o a la recolección, el análisis o la interpretación de los datos;

- redacción o revisión crítica importante del contenido del manuscrito;

- aprobación final de la versión publicada, y

- asunción de responsabilidad de la publicación.

Se consideró que todos los autores cumplían con el último criterio ya que, como parte del proceso de publicación, la revista solicita una declaración 
juramentada en la que los autores firman y se hacen responsables de todos los aspectos del manuscrito. En los casos en los que no se mencionó alguno de los criterios en la sección de contribución de autoría, se consideró que estos no se habían cumplido. Se consideró que un artículo tenía autores de regalo cuando al menos un autor no cumplía con alguno de los tres primeros criterios del International Committee of Medical Journal Editors.

Además, se estudiaron otras variables, como el número de autores por artículo y el año de su publicación.

\section{Análisis de procesamiento de datos}

Se hizo un análisis descriptivo de los datos. Las variables categóricas (cumplimiento de cada criterio de autoría del International Committee of Medical Journal Editors y presencia de autoría de regalo en el artículo original) se sintetizaron mediante frecuencias y porcentajes. La variable cuantitativa (número de autores por artículo) se sintetizó con medidas de tendencia central y dispersión, como la mediana y los rangos. Los análisis se hicieron con el software estadístico Stata ${ }^{\mathrm{TM}}$, versión 12.

\section{Aspectos éticos}

Se mantuvo la confidencialidad de los datos al no colocar el nombre de los autores ni ninguna forma de identificación de los artículos estudiados en el manuscrito.

\section{Resultados}

Se revisaron 209 artículos originales publicados desde enero de 2013 hasta marzo de 2017. Estos contaron con un total de 1.062 autores. Once $(5,3 \%)$ artículos fueron excluidos del análisis por no presentar la sección de contribuciones de autoría. La mediana del número de autores por artículo fue de 5, y el rango entre 1 y 26. De los 198 artículos incluidos, 106 (53,7 $\%)$ presentaron al menos un autor que no cumplía los criterios de autoría del International Committee of Medical Journal Editors. Se observó una tendencia a la disminución del número de artículos con autorías de regalo en los años 2016 a 2017, es decir, los dos últimos años analizados (figura 1).

En los 198 artículos incluidos hubo un total de 1.003 autores. Del total de autores, 377 (37,6 \%) no cumplieron alguno de los criterios de autoría, $112(11,2 \%)$ no cumplían el criterio de "contribuciones significativas a la concepción o diseño del manuscrito, o a la recolección, análisis o interpretación de los datos", 169 (16,8 \%) no cumplían el criterio de "redacción o revisión crítica importante del contenido del manuscrito" y 233 (23,2\%) no cumplían el criterio de "la aprobación final de la versión publicada".

\section{Discusión}

En este estudio se halló que cinco de cada diez artículos poseían, al menos, una autoría de regalo. Entre los criterios que menos se cumplían se encontraron la redacción o revisión crítica del manuscrito y su aprobación final.

En el presente estudio, la prevalencia de autoría de regalo fue de 53,7 $\%$, la cual se encuentra dentro del rango hallado en otros estudios: 17,6 a $89 \%$ de artículos originales con autorías de regalo (7-10). Así, en un estudio realizado en seis revistas de alto impacto (Journal of the American Medical Association, New England Journal of Medicine, Nature Medicine, Annals of 


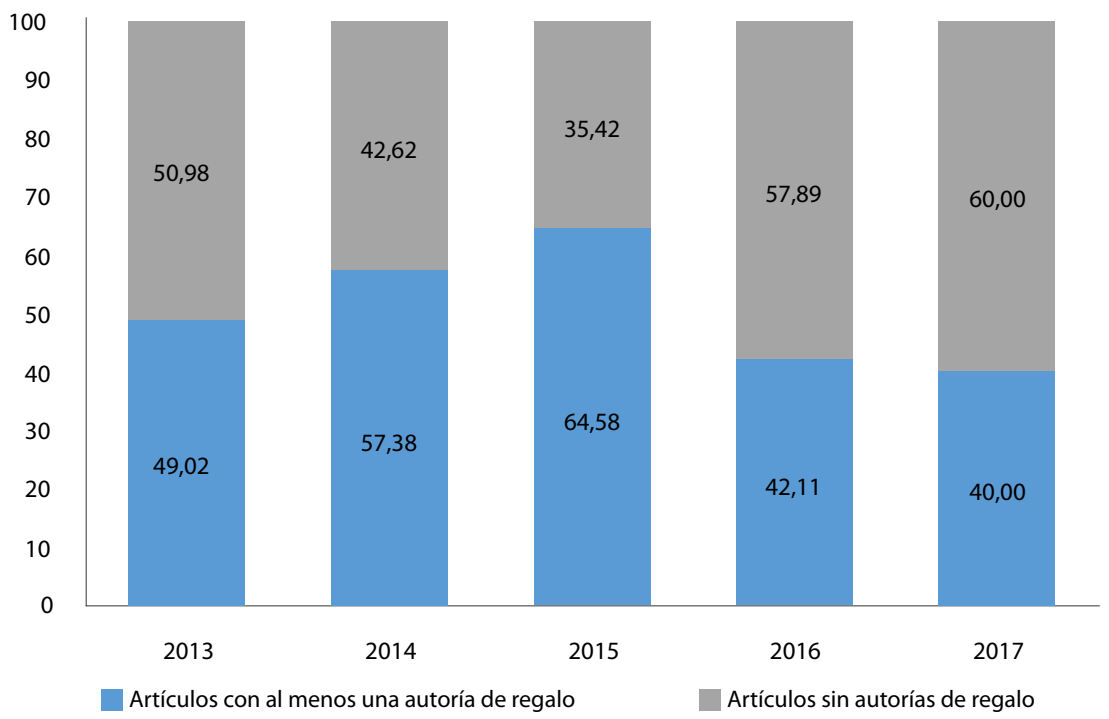

Figura 1. Proporción de artículos con autorías de regalo en los últimos cinco años

Internal Medicine, Lancet y PLoS Medicine) se halló una prevalencia de 17,6 \% (10) y, en un estudio que incluyó artículos publicados en revistas iraníes, dicha prevalencia fue de $89 \%$ (7). Sin embargo, la metodología empleada en esos estudios fue distinta a la aquí utilizada, ya que en aquellos se contrastaron las autorías de los artículos publicados con información brindada por los autores corresponsales mediante una encuesta realizada de forma virtual.

En este estudio, los criterios de autoría que no cumplían la mayoría de los autores fueron la redacción o revisión del manuscrito (17\%) y la aprobación de la versión final (23\%). Esto es similar a lo hallado en un estudio de Cochrane, en el que los criterios del International Committee of Medical Journal

Editors que menos se cumplían fueron el criterio de redacción o revisión del manuscrito (22\%) y el criterio de aprobación de la versión final (18\%) (9). Es posible que estos criterios no se cumplan debido a las múltiples modificaciones por las que pasa un manuscrito durante el proceso de publicación, en el cual es posible que los autores aprueben la versión enviada para publicación, pero no así la versión final después de las correcciones o modificaciones sugeridas por los revisores. Además, es posible que ciertos cambios mínimos realizados por el editor solo sean aprobados por el autor corresponsal.

Otros motivos por los cuales se puede incurrir en autoría de regalo, es que se invite a ser autor de un artículo a personas que desarrollan labores que podrían ser consideradas dentro del primer criterio, como reclutar sujetos de estudio, obtener financiamiento o material de estudio, supervisar o hacer ilustraciones. Estos tipos de apoyo pueden mencionarse en la sección de agradecimientos del manuscrito, mas no debe considerarse como autor al que los brinda, debido a que no ha tenido una contribución sustancial al desarrollo de la investigación (5). Además, la falta de conocimiento de los criterios de autoría podría generar autoría de regalo. En este sentido, en un estudio en Archives of Physical Medicine and Rehabilitation, se encontró que la autoría de regalo percibida fue de $18 \%$, mientras que la real, aquella obtenida siguiendo los criterios del International Committee of Medical Journal Editors, fue del $55 \%$ (17). Por otro lado, en un estudio de la revista Radiology, se informó una asociación entre incurrir en autoría de regalo y no haber tenido cursos o charlas sobre ética en investigación (18). 
En este estudio se apreció una ligera disminución de la proporción de artículos con autoría de regalo durante los últimos años evaluados. Ello podría obedecer a un mejor control de la revista al recibir los manuscritos o a un mejor conocimiento de las buenas prácticas de asignación de autoría de los investigadores.

Una limitación del presente estudio es que no se pudo comprobar si las contribuciones reportadas eran reales. Las autorías reportadas en la sección de contribuciones de autoría de la revista, podrían no ser reales debido a que:

1. Ios autores pueden tratar de adjudicar todos los criterios a todos los autores, ya que es un requisito de la revista;

2. puede existir una falla en el reporte de las contribuciones, a pesar de que esto se recalca en las instrucciones para los autores, o en la declaración juramentada que se presenta para la remisión de los manuscritos a la revista, 0

3. los editores de la revista pueden haber descartado algunos manuscritos por no cumplir con los criterios de autoría, lo cual nos indicaría que el problema es mucho mayor al hallado en este estudio.

Por estos motivos, no se puede afirmar si este estudio sobrevaloró o subestimó los valores reales de autoría de regalo. Sin embargo, consideramos que esta primera aproximación es útil, ya que refleja la magnitud del problema.

El presente estudio documenta el problema de la autoría de regalo como una práctica inadecuada llevada a cabo con frecuencia. Se recomienda a las instituciones educativas que forman investigadores que incluyan cursos o capacitación en conducta responsable. De esta manera, los investigadores estarían en capacidad de discriminar entre aquellas personas que cumplen los criterios de autoría y quienes no lo hacen. Estos últimos deberían ser nombrados en la sección de "Agradecimientos", listando sus contribuciones.

En cuanto a los editores de la revista, es recomendable solicitar al autor corresponsal que cite las contribuciones de cada autor. Esto permitiría evidenciar el grado de participación de cada autor en la investigación. Además, se recomienda que verifiquen el cumplimiento de los criterios de autoría.

En conclusión, la alta prevalencia de autoría de regalo encontrada, nos indica que es necesario tomar medidas educativas para que los investigadores asignen de manera correcta las autorías, así como medidas de control por parte de los editores de las revistas para evitar que estos problemas continúen presentándose.

\section{Agradecimientos}

Agradecemos a Liz Veramendi-Espinoza y a Fanny Tanaka-Gondo por las sugerencias para mejorar la redacción del manuscrito. Agradecemos las revisiones de los evaluadores que resultaron en mejoras sustantivas de este manuscrito.

\section{Referencias}

1. International Committe of Medical Journal Editors (ICMJE). Defining the role of authors and contributors 2018. Fecha de consulta: 28 de octubre de 2018. Disponible en: http://www. icmje.org/recommendations/browse/roles-and-responsibilities/defining-the-role-of-authorsand-contributors.html

2. Wager E. Recognition, reward and responsibility: Why the authorship of scientific papers matters. Maturitas. 2009;62:109-12. https://doi.org/10.1016/j.maturitas.2008.12.001 
3. International Committe of Medical Journal Editors (ICMJE). The New ICMJE recommendations (August, 2013). Fecha de consulta: 28 de octubre de 2018. Disponible en: http://www.icmje.org/news-and-editorials/new_rec_aug2013.html

4. Teixeira da Silva JA, Dobranszki J. Multiple authorship in scientific manuscripts: Ethical challenges, ghost and guest/gift authorship, and the cultural/disciplinary perspective. Sci Eng Ethics. 2016.;22:1457-72. https://doi.org/10.1007/s11948-015-9716-3

5. Slone RM. Coauthors' contributions to major papers published in the AJR: Frequency of undeserved coauthorship. Am J Roentgenol. 1996;167:571-9. https://doi.org/10.2214/ajr.167.3.8751654

6. Kulakowski EC, Chronister LU. Research administration and management. Sudbury, MA: Jones and Bartlett Publishers; 2008.

7. Mirzazadeh A, Navadeh S, Rokni M, Farhangniya M. The prevalence of honorary and ghost authorships in iranian bio-medical journals and its associated factors. Iran J Public Health. 2011;40:15-21.

8. Roper N, Korenstein D. Industry collaboration and primary guest authorship of high-impact randomized clinical trials: A cross-sectional study. J Gen Intern Med. 2015;30:1421-5. https://doi.org/10.1007/s11606-015-3299-1

9. Mowatt G, Shirran L, Grimshaw JM, Rennie D, Flanagin A, Yank V, et al. Prevalence of honorary and ghost authorship in Cochrane reviews. JAMA. 2002;287:2769-71. https://doi.org/10.1001/jama.287.21.2769

10. Wislar JS, Flanagin A, Fontanarosa PB, Deangelis CD. Honorary and ghost authorship in high impact biomedical journals: A cross sectional survey. BMJ. 2011;343:d6128. https://doi.org/10.1136/bmj.d6128

11. Huamaní $C$, Mayta-Tristán P. Producción científica peruana en medicina y redes de colaboración, análisis del Science Citation Index 2000-2009. Rev Peru Med Exp Salud Pública. 2010;27:315-25.

12. Scimago Lab Scopus. Scimago Journal and Country Rank. 2007-2018. Fecha de consulta: 28 de octubre de 2018. Disponible en: https://www.scimagojr.com/countryrank.php?region=Latin\%20America

13. Valenzuela G. Irregularidades en la publicación de trabajos científicos. An Fac Med. 2008:69:56-8.

14. Rao TSS, Andrade $C$. The MMR vaccine and autism: Sensation, refutation, retraction, and fraud. Indian J Psychiatry. 2011;53:95-6. https://doi.org/10.4103/0019-5545.82529

15. Burstein Z, Mayta-Tristan P. The Revista Peruana de Medicina Experimental y Salud Pública now indexed in MEDLINE/Index Medicus. Rev Peru Med Exp Salud Pública. 2010;27:312-4.

16. Revista Peruana de Medicina Experimental y Salud Pública. Instrucciones al autor 2018. Fecha de consulta: 28 de octubre de 2018.. Disponible en: http://www.rpmesp.ins.gob.pe/index.php/rpmesp/pages/view/instrucciones

17. Rajasekaran S, Shan RL, Finnoff JT. Honorary authorship: Frequency and associated factors in physical medicine and rehabilitation research articles. Arch Phys Med Rehabil. 2014;95:418-28. https://doi.org/10.1016/j.apmr.2013.09.024

18. Eisenberg RL, Ngo LH, Bankier AA. Honorary authorship in radiologic research articles: Do geographic factors influence the frequency? Radiology. 2014;271:472-8. https://doi.org/10.1148/radiol.13131710 